\title{
THE HIERARCHY MODEL OF THE SIZE DISTRIBUTION OF CENTRES
}

\section{by J. Tinbergen*}

\section{INTRODUCTION}

We know that human beings live in centres, that is, cities, towns and villages of different size. Both large and small centres have a number of advantages and disadvantages, different for different people and this is why we have a whole range of sizes. Statistically, we even find that the size distribution is fairly regular. No scientific explanation worthy of that name has been advanced so far. Neither do we know whether or not the existing distribution is optimal.

Not all forces at work are economic forces. Yet economic forces seem to play an important role. Economic theory so far has neglected the subject. An explanation of the size distribution requires the introduction of location and hence of transportation and communication into economic theory. This constitutes an enormous complication because of the large increase in the number of variables which a full-fledged introduction of the these elements would require. Therefore, any theory of the economics of space has to start out with simplifying assumptions.

The model to be presented and discussed here is an example. In fact, it only constitutes a hypothesis, hanging in the air, so to say, between theory and observation. That is, it cannot be proven theoretically to be correct and it cannot be proven that it reproduces either reality or an optimum situation. It may serve as a starting point for both more refined theoretical models and empirical verification. In this paper, an endeavour is made to present four modest contributions: in Section 2, we briefly summarize a model to be called the simplest version of a hierarchy model; in Section 3, we indicate some cases in which the model actually constitutes an optimum size distribution; in Section 4, we describe some possibilities for generalizing the model and in Section 5, we discuss one empirical test.

\section{SIMPLEST VERSION OF THE HIERARCHY MODEL}

We assume a closed country (i.e., a country without foreign trade) having a regular form, say a square, which is evenly covered with agricultural production units except in the centres. We further assume an arbitrary number of industries, $H$, each producing finished products, indicated by a number $h$, where $h=0,1, \ldots, H$. We call $h$ the rank of the industry considered. The case where $h=0$ represents agriculture. For each product, a given size of enterprise

\footnotetext{
* The author is associated with the Netherlands School of Economics, Rotterdam, Netherlands.
} 
exists, supposedly the optimal. The number of such enterprises needed to satisfy the demand for product $h$ is indicated by $n_{h}$, and is derived from total demand for the product which is equal to $\alpha_{\hbar} Y$, where $Y$ is the country's income and $\alpha_{h}$ is a given demand ratio for product $h$. The industries have been ordered in such a way that $n_{1} \geqq n_{2} \geqq n_{3} \ldots \geqq n_{H}$ and we assume also that $n_{H}=1$. Since we assume that all income is spent,

$$
\alpha_{0}+\alpha_{1}+\alpha_{2}+\cdots \alpha_{H}=1 \text {. }
$$

The hypothesis about the size distribution of centres is characterized by the following sub-hypotheses.

a) There are only $H$ types of centre and these are indicated by a figure $h^{\prime}$ running from 1 to $H$, signifying the rank of each type of centre.

b) In any centre of rank $h^{\prime}$, only the industries appear for which $h \leqq h^{\prime}$. Thus, a centre of rank 5 possesses only industries: 1, 2, 3, 4 and 5 .

c) The number of enterprises in each industry in each centre is just sufficient to satisfy local demand for the industries of a rank lower than the centre's rank.

d) The industry of $\mathrm{rank} h^{\prime}$ in a centre of rank $h^{\prime}$ not only satisfies local demand, but also the demand for that product in the centres of lower rank. These exports are equally distributed among all centres of the same type $h^{\prime}$.

From the sub-hypotheses enumerated, we can derive the number of centres $n^{h^{\prime}}$ of a given rank $h^{\prime}$ and the total income earned $Y^{h^{\prime}}$ in all centres of that rank. The formulae can be written in the following form:

$$
\begin{aligned}
Y^{0} & =\alpha_{0} Y ; \\
Y^{0}+Y^{1} & =\frac{\alpha_{0} Y}{1-\alpha_{1}} ; \\
Y^{0}+Y^{1}+Y^{2} & =\frac{\alpha_{0} Y}{1-\alpha_{1}-\alpha_{2}} ;
\end{aligned}
$$

and so on;

or in general:

$$
Y^{0}+Y^{1}+\cdots Y^{h}=\frac{\alpha_{0} Y}{1-\alpha_{1}-\alpha_{2} \cdots \alpha_{h}}=\frac{\alpha_{0} Y}{\alpha_{h+1}+\alpha_{h+2}+\cdots \alpha_{H}+\alpha_{0}}
$$

from which we see, incidentally, that the total income for all types of centre adds up to $Y$. We can also calculate the number of centres of each rank, if we add the assumption that there is always only one enterprise of the highest rank in each centre. For the number $n^{h^{\prime}}$, we find:

$$
n^{h^{\prime}}=n_{h^{\prime}} \frac{\alpha_{0}}{1-\alpha_{1} \cdots-\alpha_{h^{\prime}}} .
$$

Formulae (5) and (6) determine the size distribution of centres. In addition the number of enterprises of rank $h$ in all centres $h^{\prime}$ will be found to be:

$$
n_{h}^{h^{\prime}}=\frac{\alpha_{0} \alpha_{h^{\prime}} n_{h}}{\left(1-\alpha_{1}-\alpha_{2} \cdots-\alpha_{h^{\prime}-1}\right)\left(1-\alpha_{1}-\alpha_{2} \cdots-\alpha_{h^{\prime}}\right)} .
$$

\section{CASES IN WHICH A PROOF OF OPTIMALITY CAN BE GIVEN}

The model shown constitutes an optimum (in the sense of minimizing total 
transport costs of all products transported from one centre to another) only if certain conditions are fulfilled. These conditions have been explicitly formulated in a few simple cases only. One example is given by the author and is characterized by $H=2$. This example involves some different assumptions about the geometrical location of the centres, as well as about the nature of transportation costs. ${ }^{1}$ With regard to the latter, three possibilities have been considered:

i) transport costs do not depend on distance nor on the return freight, but only on the type of good transported;

ii) these transport costs still do not depend on distance, but of the two opposite flows of transportation, only the "dominant flow" (the larger of the two) determines the costs, again depending on the type of good transported;

iii) in addition to the assumptions made under (ii), the transport costs now also depend on distance.

Under assumptions (i) and (ii), the simple version of the hierarchy model represents the optimum distribution of centres for $H=2$, irrespective of their geometrical location. Under assumption (iii), the simple version constitutes the optimum only if additional assumptions are fulfilled.

More cases have been analyzed by Bos who also introduced other methods of research. ${ }^{2}$ For instance, in one method, a finite number of locations for each enterprise is assumed to exist: in another, production costs are assumed to depend on the size of the enterprise.

\section{POSSIBILITIES OF GENERALIZATION}

Calculation of the size distribution, as shown for the simplest version, remains possible in a number of other versions of the hierarchy model. It should be added that this does not necessarily mean that a proof of optimality, as indicated in Section 3, can also be given. Further research is needed on this subject. Other versions can be constructed without difficuly in which:

a) some types of foreign trade occur;

b) not only final products, but intermediate products are produced;

c) a particular industry (such as 'mining' or 'port activities') can operate at one location only;

d) exports from any type of centre to other types of centre include not only the products of the highest rank, but products of lower rank also.

\section{AN EMPIRICAL TEST}

This section gives the first results of an attempt to test the hierarchy hypothesis using French data for $1962 .^{3} \quad$ For several reasons related to the geometrical interpretation of the hypothesis and to transport efficiency, it seems probable that the optimal ratio of the number of centres of a given rank to the number of centres of the next higher rank is equal to four. Applying this ratio and using population and per capita income data, the demand coefficients

1 J. Tinbergen, "Sur un Modèle de la Dispersion Géographique de l'Activité Economique," Revue d'Economie Politique, Vol. 74 (January-February 1964), p. 30. (Reprint Series of the Division for Balanced International Growth of the Netherlands Economic Institute, No. 22.)

${ }^{2}$ H. C. Bos, Spatial Dispersion of Economic Activity (Rotterdam: Rotterdam University Press, 1965).

${ }_{3}^{3}$ This verification for France has been undertaken by Mr. L. B. M. Mennes, M. A. 
$\alpha_{h}$ and the number of technical units in each industry $n_{h^{\prime}}$ have been calculated by means of equations (2) through (6). In this way, seven ranks have been distinguished while the ratio of the number of technical units of an industry of a given rank to the number of units of an industry of the next higher rank turned out to be approximately five.

Next, a frequency distribution of all French industries according to the number of technical units has been drawn up. Apart from the number of technical units, the number of workers in each industry is also given in this distribution. Using these data on the number of workers by industry in order to estimate $\alpha_{h}$ values and applying the ratio of five, mentioned above, the number of centres of each rank and the population living in each type of centre have been calculated, again by means of equations (2) through (6). This theoretical distribution of population and the actual distribution are shown in Table 1.

TABLE 1. Actual and Theoretical Distributions of Population in Thousands of Persons

\begin{tabular}{|c|r|r|r|}
\hline Rank & $n^{h^{\prime}}$ & $\begin{array}{c}\text { Theoretical } \\
\text { Population }\end{array}$ & $\begin{array}{c}\text { Actual } \\
\text { Population }\end{array}$ \\
\hline 7 & 1 & $5,100.8$ & $7,813.9$ \\
6 & 4 & $3,575.9$ & $2,626.8$ \\
5 & 20 & $10,040.9$ & $4,725.3$ \\
4 & 69 & $9,335.8$ & $5,317.0$ \\
3 & 203 & $5,507.8$ & $4,615.3$ \\
2 & 599 & $1,959.7$ & $3,671.7$ \\
1 & 2257 & $1,314.9$ & $5,174.9$ \\
0 & & $10,722.6$ & $13,613.5$ \\
\hline
\end{tabular}

The two distributions are rather different from each other. Except for the centre of the highest rank, Paris, the hierarchy model predicts a much higher concentration of population in the higher ranked centres than actually is the case. For the centres lower rank, the opposite is true. In these centres, the number of people actually present is much higher than the hierarchy model indicates. It remains an open question, of course, whether the deviation means nonoptimality in the actual distribution or lack of realism in the theoretical one. Moreover, these results are preliminary and further experiments with the model are still in the process of being carried out. 